IV. Ueber den Zusammenhang der electromagnetischen Rotation mit der unipolaren Induction; von E. Edlund.

\$. 1 .

$\mathbf{W}_{\text {ir wollen uns einen cylindrischen Stahlmagnet denken, }}$ der um seine geometrische Axe rotiren kann und von einem um dieselbe Axe drehbaren Metallmantel umgeben ist, und annehmen, dass das eine Ende eines ruhenden Leitungsdrahtes den Mantel in der Nähe des einen Magnetpols und das andere in einem zwischen den beiden Polen liegenden Punkt berührt. Wenn dann ein galvanischer Strom von hinreichender Stärke in den Draht hineingeführt wird, so wird bekanntlich der Mantel während der Dauer des Stromes in Rotation gesetzt. Wenn der Strom umgekehrt wird, so verändert auch die Rotation ihre Richtung. Weil es für das Folgende von Wichtigkeit ist, mag es hier schon bernerkt werden, dass der Magnet selbst, wie beweglich er auch sei und wie gross die Stromstärke auch genommen werde, nicht in Rotation gesetzt'wird. Der Magnet beginnt erst dann zu rotiren, entweder wenn er mit dem Mantel fest verbunden wird, in welchem Falle er von dem rotirenden Mantel mitgeschleppt wird, oder wenn der Mantel weggenommen und der Leitungsdraht auf die oben angegebene Weise mit dem Magnet galvanisch verbunden wird, in welchem Falle der Magnet als Leiter denselben Dienst wie der Mantel in dem vorigen Falle leistet.

Es ist klar, dass die beobachtete Rotation durch die Einwirkung des Magnetes auf den in dem beweglichen Leiter laufenden Strom verursacht wird; dieser bewegliche Leiter mag nun aus dem Mantel oder dem Magnet selbst bestehen. Ferner kann die Resultirende der zwischen dem Magnet und dem in dem Mantel laufenden Strome stattfindenden Wechselwirkung unter allen Verbältnissen un- 
möglich durch die Axe des Magnets gehen. Es wäre also zu erwarten, dass der Magnet selbst durch diese Wechselwirkung in eine derjenigen des Mantels entgegengesetzte Rotation gesetzt würde. Dieses würde auch geschehen, wenn nicht zugleich eine Wechselwirkung zwischen dem Magnet und der festen Strombahn stattfände, welche dem Magnet eine gleich grosse, aber entgegengesetzte Rotation zu ertheilen suchte. Dass der Magnet während der Rotation des Mantels still steht, beweist also in der That, dass die Wechselwirkungen der beiden Stromtheile auf den Magnet gleich gross sind, aber eine entgegengesetzte Richtung haben.

Wenn der Mantel rotirt, so verrichtet die electromagnetische Wechselwirkung zwischen dem Magnet und dem Strom in dem Mantel eine mechanische Arbeit, welche unter übrigens gleichen Verhältnissen in einer gewissen Zeit dem Product aus dem magnetischen Moment $M$ des Magnets, der Stromstärke $s$ in dem Mantel und dem Winkel $w$, den der Mantel in derselben Zeit beschreibt, proportional ist. Diese Arbeit laann mithin durch $k M s w$ ausgedrückt werden, wo $k$ eine ron der Beschaffenheit des Mantels und den Lagen der Punkte, wo die übrige Leitung den Mantel berülırt, abhängige Constante ist. Wenn ferner dieses Product mit dem Wärmeäquivalent $(A)$ multiplicirt wird, so erhält man als Ausdruck für den Wärmewerth der verrichteten Arbeit $A k M s w$.

Wenn der Mantel während des Durchganges des Stromes durch eine äussere Kraft zu rotiren verhindert wird, so besteht die ganze Wirkung des Stromes darin, dass er in der Leitungsbahn Wärme erzeugt. In derselben Zeit aber wird Wärme ron der electromotorischen Kraft rerbraucht, und weil dieser Würmeverbrauch der von dem Strom in der Leitungsbahn erzeugten Wärmemenge gleich ist, so wird die ganze ron dem Strom und der electromotorischen Kraft hervorgebrachte Wärmeveränderung gleich Null. Die durch die chemischen Processe in der Säule 
hervorgerufene Wärmemenge muss als das Wärmeäquivalent dieser Processe betrachtet werden, und würde dieselbe Grösse erreicht haben, wenn diese chemischen Processe ohne Erzeugung eines galvanischen Stromes stattgefunden hätten. Wie ich in einer früheren Arbeit gezeigt habe, führen alle Versuche von Favre, Raoult und anderen über die Wärmeverhältnisse in der Säule und deren Leitungen $z \mathfrak{u}$ diesem Resultat, dessen Richtigkeit übrigens auf theoretischem Wege bewiesen werden kann. ${ }^{1}$ ) Dieses Resultat muss folglich als vollkommen zuverlässig betrachtet werden. Wenn $E$ die electromotorische Kraft, $l$ den ganzen Leitungswiderstand, $m$ eine Constante und $s$ die Stromstärke in diesem Falle bezeichnen, so wird die von der electromotorischen Kraft verbrauchte Wärmemenge durch $m E s$ und die durch den Durchgang des Stromes durch die Leitung hervorgebrachte Wärmemenge durch $m s^{2} l$ ausgedrückt. Weil aber $s=\frac{E}{l}$ ist, so sind diese -beiden Ausdrücke gleich gross, und die ganze Wärmeproduction also gleich Null. Wenn der Mantel während des Durchganges des Strones rotirt, so wird von dem Strom eine gewisse mechanische Arbeit verrichtet, und es folgt dann aus den Principien der mechanischen Wärmetheorie, dass die Wärmeproduction des Stromes eine dieser Arbeit entsprechende Verminderung erleiden muss. Dieselbe kann nur auf eine einzige Weise erreicht werden, nämlich durch die Entstehung eines Stromes durch Rotation des Mantels, der in entgegengesetzter Richtung wie der die Rotation hervorbringende Strom fliesst. Wenn dieser Strom $s_{1}$ genannt wird, ist folglich der ganze durch die Leitung fliessende Strom gleich $s-s_{1}$. Weil die ganze Wärmeproduction in dem Falle, dass der Mantel festgehalten wurde, gleich Null war, so muss nun ein Wärmeverlust entstehen, der ausgedrückt wird durch:

1) Pogg. Ann. CLIX. p. 420; Phil. Mrag. (5). III. p. 428. (1877). 


$$
\begin{gathered}
+m\left(s-s_{1}\right)^{2} l-m E\left(s-s_{1}\right), \text { oder weil } s=\frac{E}{l} \\
\text { durch }-m l s_{1}\left(s-s_{1}\right) .
\end{gathered}
$$

Weil dieser letzte Ausdruck, mit verändertem Vorzeichen genommen, dem Wärmewerth der verrichteten Arbeit gleich sein muss, so erhält man hieraus die Gleichung:

$$
A k M\left(s-s_{1}\right) u=m l s_{1}\left(s-s_{1}\right),
$$

oder, wenn $p$ der Kürze wegen eine neue Constante bedeutet:

$$
l s_{1}=p M w .
$$

Durch die Rotation des Mantels entsteht folglich ein Strom, der dem die Rotation rerursachenden Strome entgegengesetzt gerichtet ist, und dessen Intensität dem magnetischen Momente direct und dem Leitungswiderstande umgekehrt proportional ist. Der Ausdruck für $s_{1}$ ist nur in soweit von der die Rotation hervorbringenden Stromstärke abhängig, als diese die Grösse ron $w$ bestimmt.

Die mechanische Wärmetheorie fordert also mit Nothwendigkeit, dass die Rotation des Mantels ron der Entstehnung eines Stromes begleitet ist. Dabei ist es ganz gleichgültig, welcherlei Kraft die Rotation rerursacht, ob sie eine electromagnetische oder äussere mechanische sei. Wenn also die Säule aus der Leitungsbahn entfernt, und der Mantel durch eine äussere mechanische Kraft in $\mathrm{Ro-}_{0}$ tation gesetzt wird, so muss ein galvanischer Strom von solcher Richtung entstehen, dass er den Mantel in entgegengesetzter Richtung zu drehen sucht.

Der Widerstand, den die mechanische Kraft, um den Mantel dauernd in Rotation zu erhalten, zu überwinden hat, ist von zweierlei Art: erstens besteht er aus allen äusseren Hindernissen, wie Reibung, Luftwiderstand etc. und zweitens aus der electromagnetischen Wechselwirkung zwischen dem Magnet und dem Strom, welche den Mantel in entgegengesetzter Richtung zu drehen sucht. Der erste Theil dieser Arbeit wird in Wärme verwandelt und hat folglich mit der Strombildung nichts zu thun. Der zweite 
Theil dagegen wird die Ursache des electrischen Stromes. Es kann mithin die Grösse dieser Reaction mit $k M s_{1} w$ und deren Wärmewerth mit $A k M s_{1} w$ ausgedrückt werden. Diese Wärmemenge muss derjenigen, die der entstandene Strom in der ganzen Leitungsbahn erzeugt, gleich sein, und man erhält die Gleichung:

$$
A k M s_{1} w=m s_{1}{ }^{2} l \text {, oder } l s_{1}=p M w .
$$

Das Gesetz der Strombildung wird also das nämliche, wie wenn ein galvanischer Strom die Rotation hervorgebracht hätte. Es verdient hierbei bemerkt zu werden, dass die electromotorische Kraft von dem Leitungswiderstande des Mantels unabhängig ist.

Wie oben angegeben wurde, wird der Magnet nicht in Rotation gesetzt durch einen Strom, der den Mantel und die feste Leitungsbahn durchläuft. Die Erfahrung hat gelehrt, dass ein geschlossener Strom nicht im Stande ist einen Magnet in Rotation zu bringen, mag er innerhalb oder ausserhalb der Leitungsbahn gelegen sein oder von derselben auf irgend eine Weise geschnitten werden, so dass ein Theil des Magnets sich ausserhalb und der andere innerhalb der Strombahn befindet. Die Ursache davon, dass der Magnet rotirt, wenn er einen Theil der Leitungsbahn ausmacht, ist keine andere als die, welche den Magnet bei dem bekannten Plücker'schen Versuche in Rotation bringt, wenn der Magnet mit dem Mantel fest verbunden ist. In beiden Fällen wird der Magnet von dem rotirenden Leiter mitgeschleppt. Das Drehungsmoment des ganzen Stromes auf den Magnet ist gleich Null, und dieser Satz behält seine Gültigkeit auch in dem Falle, dass den Magnet als Stromleiter dient. Dies folgt übrigens aus der schon früher gemachten richtigen Annahme, dass das Drehungsmoment des einen Theils des geschlossenen Stroms auf den Magnet dem des anderen vollkommen gleich ist, nur dass beide verschiedene Vorzeichen haben. Wenn man also die Säule aus der Leitungsbahn wegnimmt, und mit Hülfe einer äusseren mechanischen Kraft den Magnet in Ro- 
tation bringt, so kann dabei kein Inductionsstrom entstehen, weil das Drehungsmoment des entstandenen Inductionsstroms auf den Magnet gleich Null sein würde. Die äussere mechanische Kraft hätte in diesem Falle keinen electromagnetischen Widerstand zu überwinden; es ist aber gerade die für das Ueberwinden dieses Widerstandes verbrauchte Arbeit, die den Strom verursacht. Um einen electrischen Strom zu bilden, wird entweder Wärme oder mechanische Arbeit verbraucht; aus nichts kann natürlich der Strom nicht entstehen. Die bisher geltende, von mir aber schon früher bestrittene ${ }^{1)}$ Annahme, dass der unipolare Inductionsstrom durch die Rotation des Magnets verursacht wird, kann also nicht richtig sein. Auch wenn der Magnet selbst einen Theil der Leitungsbahn ausmacht, entsteht der Inductionsstrom nicht dadurch, dass der Magnet als Magnet in Rotation gebracht wird, sondern aus dem Grunde, dass ein Theil der Leitungsbahn rotirt. Eine Theorie, die zu einem entgegengesetzten Resultat führt, kann unmöglich richtig sein und ist folglich für die Erklärung dieser Erscheinungen unbrauchbar.

Aus dem Angeführten dürfte hervorgehen, dass der natürliche Zusammenhang zwischen der electromagnetischen Rotation und der unipolaren Induction mit Beihülfe der Principien der mechanischen Wärmetheorie sich leicht nachweisen lässt. Es ist hierbei ganz gleichgültig, welche Ansicht man sich von der Natur der Electricität gebildet hat. Um eine Naturerscheinung vollständig zu erklären, ist es aber keineswegs hinreichend, ihre nothwendige Verbindung mit einer anderen nachweisen zu können. Dazu wird auch erforderlich, dass man die Mittel anzugeben vermag, die die Natur anwendet, um die fragliche Erscheinung hervorzubringen. Ich will deswegen im Folgenden zu erörtern suchen, auf welche Weise die unipolaren Inductionserscheinungen entstehen.

1) Pogrg. Aun. CLVI. p. ว90. CLVII. p. 630. 


\section{§. 2.}

Wir denken uns einen senkrecht stehenden Magnet, dessen Nordpol nach oben gerichtet ist. Wenn dieser Magnet durch ein Solenoid ersetzt werden soll, so läuft in diesem der Strom, von oben angesehen, gegen die Zeiger einer Uhr. Ringsum den Magnet oder das Solenoid denken wir uns ferner einen kreisförmigen linearen, leicht zu hebenden und zu senkenden Leiter, dessen Ebene horizontal ist und also rechtwinklig auf der Axe des Magnets oder Solenoids steht. Wenn nun der Leiter zwischen dem einen oder anderen Pol und der Indifferenzebene des Magnets gelegen ist, und man durch denselben einen Strom in entgegengesetzter Richtung gegen die Zeiger einer Uhr oder wie der Strom des Solenoids leitet, so sucht der Magnet den Stromring stets gegen die Indifferenzebene hinzuführen, wo er eine stabile Gleichgewichtslage erreicht. Wenn der Strom, von oben angesehen, in derselben Richtung wie die Zeiger einer Uhr den Leiter durchläuft, so entsteht ein anderes Verhältniss. Der zwischen der Indifferenzebene und dem einen oder anderen Pol gelegene Leiter sucht sich dem nächstliegenden Pol zu nähern. Wenn er in derselben Höhe wie die Indifferenzebene liegt, so befindet er sich in labilem Gleichgewicht. Ist also der Magnet oder das Solenoid längs seiner ganzen Höhe von solchen leichtbeweglichen Stromringen umgeben, so werden diese, wenn die Ströme gegen die Zeiger einer Uhr gehen, sich in der Indifferenzebene sammeln; wenn aber die Ströme die entgegengesetzte Richtung haben, so wird ein 'Theil der Stromringe dem Nordpol und der andere Theil dem Südpol sich nähern. Ist der Südpol des Magnets nach oben gerichtet, so tritt das entgegengesetzte Verhalten ein. Die Kraft, mit der die Stromringe bewegt werden, ist unter übrigens gleichen Verhältnissen dem Product aus dem magnetischen Momente und der Stromstärke proportional.

Obgleich die Richtigkeit des eben Angeführten einAnv. d. Phys, u. Chem. N. F. Ir. 
leuchtend ist, habe ich doch dieselbe noch durch experimentelle Versuche bestätigt, die indess so einfach und leicht anzustellen sind, dass eine nähere Beschreibung überflüssig sein dürfte.

Wir denken uns einen senkrechten hohlen Metallcylinder und in diesem einen Eisencylinder. Wird der Eisencylinder auf die eine oder andere Weise magnetisch gemacht, so entstehen dabei in dem Metallcylinder Inductionsströme, welche in horizontaler Richtung den Eisenkern umkreisen. Die freien Aethermolecüle werden von der inducirenden Magnetkraft aus den Gleichgewichtslagen. die sie vor der Magnetisirung besassen, fortgeführt; dieselbe inducirende Kraft wirkt aber auch auf die gebundenen Aetherschichten, von welchen die eigenen Molecüle des Leiters umgeben sind. Die Dichtigkeit dieser Aetherschichten wird demnach auf der Seite grösser, welche gegen den Aetherstrom gewandt ist. Diese Aetherschichten üben natürlich eine Repulsionswirkung auf die freien, sich bewegenden Aethermoleciule aus, und die Resultirende dieser Repulsion sucht, weil sie in entgegengesetzter Richtung gegen den freien Aetherstrom wirkt, die Geschwindigkeit der freien Aethermolecüle zu vermindern. Wenn die Dichtigkeit der Aetherschichten auf der Seite, diẹ gegen den Aetherstrom gewendet ist, hinreichend gewachsen ist, wird die dadurch entstehende Repulsion auf den freien Aether so gross, dass dieser in Ruhe kommt. Wenn danach die Inductionskraft weggenommen wird, so werden die freien Aethermolecüle durch die Repulsion der verdichteten Aetherschichten zurückgetrieben, und man erhält dadurch einen Inductionsstrom, der mit dem vorigen gleich gross ist, aber eine entgegengesetzte Richtung hat. Nach dieser Ansicht besteht folglich der Inductionsstrom in dem Uebergange des Aethers aus der einen Gleichgewichtslage in die andere. ${ }^{1}$ ) Während der Magnet in dem Metallcylinder steht, werden also die Aethermolecüle in

1) Théorie des phénomènes électriques. 
diesem von zwei horizontalen, einander entgegenwirkenden Kräften in Gleichgewicht gehalten.

Wir nehmen nun an, dass der Nordpol des Magnets nach oben gewendet ist, und wollen den ihn, umgebenden Metallcylinder mit constanter Geschwindigkeit, von oben angesehen, entgegengesetzt gegen die Zeiger einer Uhr rotiren lassen, so dass er in einer gegebenen Zeit den Winkel $w$ beschreibt. Die freien Aethermolecüle im Metallcylinder, welche auf die oben angegebene Weise verhindert werden sich in der Horizontalebene ron selbst zu bewegen, müssen dann die eigenen Molecüle des Metallcylinders begleiten und rotiren also mit derselben Geschwindigkeit wie diese. Der Leitungswiderstand des Cylinders übt hierauf keinen Einfluss aus. In der That bilden also die Aethermolecüle einen Strom von der angegebenen Richtung, dessen Stärke unter übrigens gleichen Verhältnissen mit $w$ proportional ist. Nach dem oben Angeführten aber sucht der Magnet diese Ströme der Indifferenzebene zu nähern, und die Kraft, womit dieses geschieht, ist dem Producte aus dem magnetischen Momente und der Stromstärke proportional. Sie kann folglich durch $k M w$ ausgedrückt werden, wo $k$ eine Constante ist. Hieraus folgt, dass die Dichtigkeit des Aethers in der Indifferenzebene am grössten wird, von wo aus sie stetig gegen die beiden Pole abnimmt. Wenn also ein festliegender Leitungsdraht mit dem einen Ende den Cylinder in der Indifferenzebene und mit dem anderen in der Nähe des einen Pols berührt, so bekommt man einen electrischen Strom, der in dem Draht von dem ersten zu dem letzten Ende fliesst. Wenn der ganze Leitungswiderstand $l$ genannt wird, so wird die Stromstärke $s_{1}=\frac{k M w}{l}$. Geht die Rotation in entgegengesetzter Richtung, so sammelt sich der Aether an den beiden Polen, und man erhält dadurch einen Strom, der im Draht von den Polen zu der Indifferenzebene läuft. Wenn die beiden Enden des Drahts den Cylinder in zwei Punkten berühren, die in Bezug auf die Indifferenzebene 
eine symmetrische Lage haben, so muss natürlich die Stromstärke gleich Null sein. Hat der Magnet seinen Südpol nach oben, so sammelt sich der Aether in der Indifferenzebene, wenn die Rotation, von oben angesehen, wie die Zeiger einer Uhr geht; wenn aber die Rotation eine entgegengesetzte ist, so geschitht die Ansammlung an den beiden Polen. Der Strom verändert also seine Richtung, wenn der Magnet umgewandt wird. Es ist leicht einzusehen, dass ein Solenoid, wie Forssman und Z euner bewiesen, ${ }^{1}$ ) sich in dieser Hinsicht wie ein Magnet verhalten muss. Mit einem Worte, die Thatsachen, die man auf experimentellem Wege gefunden, werden durch diese Anschauungsweise vollständig erklärt.

In dem Vorhergehenden ist nur ein Fall der unipolaren Induction behandelt worden. Man überzeugt sich aber sehr leicht davon, dass die übrigen Inductionsfälle auf dieselbe Art behandelt werden können; man braucht nämlich nur die Einwirkung des Magnets auf die durch die Rotation gebildeten Ströme in Betracht zu ziehen. Es dürfte deswegen überflüssig sein, auf dieselben hier näher einzugehen.

Stockholm, den 15. Juli 1877.

V. Ueber die von Herrn Dr. Kerr gefundene neue Beziehung zwischen Licht und Electricität; von J. J. Mackenzie.

\section{$\mathbf{U}_{\mathrm{m}}$}

$m$ die interessanten Untersuchungen ron Dr. Ker. ${ }^{2}$ ) weiter zu verfolgen und zu ermitteln, warum es dem Herrn

1) Ofvers. k. Vetensk. Förhandl, 1877 April. Pogg. Ann. CLX. p. 604 .

2) Phil. Mag. (4) Vol. L. 1875. 